\title{
Diagnóstico de animais ilegais recebidos no centro de triagem de animais silvestres de Belo Horizonte, Estado de Minas Gerais, no ano de 2011
}

\author{
Diagnosis of illegal animals received at the wildlife rehabilitation center of Belo Horizonte, \\ Minas Gerais State, Brazil in 2011
}

\author{
Ana Cláudia Parreiras de Freitas $^{\mathrm{I}^{*}}$ Misael Enrique Oviedo-Pastrana ${ }^{\mathrm{I}}$ \\ Daniel Ambrózio da Rocha VilelaII Pedro Lúcio Lithg Pereira ${ }^{I}$ \\ Lucas de Oliveira Carneiro Loureiro' João Paulo Amaral Haddad \\ Nelson Rodrigo da Silva Martins ${ }^{I}$ Danielle Ferreira de Magalhães Soares ${ }^{I}$
}

RESUMO

$O$ centro de triagem de animais silvestres em Belo Horizonte (CETAS-BH) é um dos órgãos responsáveis pelo recebimento de animais silvestres em Minas Gerais (MG). $O$ conhecimento da fauna mantida ilegal é ferramenta importante para a conservação dos animais silvestres, pois permite o aprimoramento da educação ambiental e das ações fiscalizadoras. Objetivou-se diagnosticar as espécies da fauna silvestre apreendidas ou entre gues voluntariamente no CETAS-BHe analisar espacialmente sua distribuição. As espécies foram identificadas e classificadas de acordo com a chave taxonômica e o risco de extinção. $\mathrm{Na}$ análise descritiva espacial, foram identificadas as coordenadas geográficas dos municípios dos animais para a construção dos mapas de distribuição e de densidade de Kernel. No ano de 2011, foram recebidos 7.426 animais vivos, dos quais $91,5 \%$ eram aves, $7 \%$ répteis e 1,5\% mamiferos. Verificou-se ampla variedade de espécies (166), correspondendo às aves a maioria (79,5\%). As espécies mais recebidas foram Sicalis flaveola e Saltator similis. Do total de espécies recebidas, $15 \%$ estavam ameaçadas de extinção. A principal procedência foi apreensão $(82,7 \%)$ e, delas, $79 \%$ foram realizadas pela Polícia Militar do Meio Ambiente. Os animais eram de 94 municípios de MG. A análise espacial identificou a Região Metropolitana de BH como o local de maior concentração de ocorrências. A partir dos resultados obtidos, espera-se o aprimoramento e a intensificação das ações de educação ambiental e de fiscalização para essas áreas especificas.

Palavras-chave: CETAS, distribuição espacial, fauna silvestres.

\section{ABSTRACT}

The wildlife rehabilitation center in Belo Horizonte (CETAS - BH) is one of the responsible for the receipt of wild animals in Minas Gerais (MG). The knowledge about the animals traffic is an important instrument for the wild species conservation as it allows the improvement of environmental education and the enforcement actions. The research aimed to diagnose the species of wildlife seized or voluntarily surrendered in CETAS-BH and analyze their spatial distribution. The species were identified and classified according to the taxonomic key and the risk of extinction. Descriptive spatial analysis identified the geographic coordinates of the cities where the animals were found to make maps showing their spatial distribution and the Kernel density. In 2011 were received 7426 live animals, $91.5 \%$ of these birds, $7 \%$ reptiles and $1.5 \%$ mammals. It was found a range of species (166), most of them birds (79.5\%). The most received species were Sicalis flaveola and Saltator similis. Fifteen percent of the species were endangered. The main category of receiving was the seizure (82.7\%), and $79 \%$ of them were conducted by Environment Police. The animals were from 94 cities in the state of Minas Gerais. The spatial analysis identified the Belo Horizonte Metropolitan Region as the region with the highest concentration of occurrences. In this context, this study suggests the improvement and intensification of environmental education and supervision in those specific areas.

Key words: CETAS, spatial distribution, wild animals.

\section{INTRODUÇÃO}

O estado de Minas Gerais (MG) possui grande extensão territorial, expressiva disponibilidade de recursos hídricos e a presença dos biomas Mata Atlântica, Cerrado e Caatinga. Essa situação confere ao estado uma extraordinária abundância de espécies, que contribui para a classificação do Brasil entre os cinco países com maior biodiversidade no mundo (SANTOS \& CÂMARA, 2002; BIODIVERSITAS, 2007). Desde os tempos coloniais (RIBEIRO \& SILVA, 2007), essa biodiversidade vem sendo

IEscola de Veterinária (EV), Universidade Federal de Minas Gerais (UFMG), Avenida Antônio Carlos, 6627, CP 567, 30161-970, Belo Horizonte, MG, Brasil. E-mail: anaclaudiaparreiras@yahoo.com.br. *Autor para correspondência.

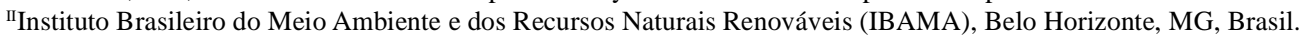


explorada de forma desordenada e predatória, expondo incessantemente os animais silvestres a vulnerações e a ameaças de extinção. Atualmente, fatores como o desmatamento, a degradação e a fragmentação de habitat, as perturbações antrópicas e a captura excessiva de animais silvestres têm intensificado as ameaças à fauna silvestre e expondo, continuamente, mais animais à extinção na natureza. $\mathrm{O}$ estado de MG foi o que contribuiu com o maior número de espécimes capturadas em todo o Brasil nos anos de 2005 a 2008 (DESTRO et al., 2012).

O Instituto Brasileiro de Meio Ambiente e dos Recursos Renováveis (IBAMA) é uma autarquia federal responsável por executar as políticas públicas do meio ambiente e de fiscalização ambiental. Para que os animais silvestres apreendidos tivessem adequada identificação, tratamento, triagem e destino, foram criados os centros de triagem de animais silvestres (CETAS), mantidos pelo IBAMA a partir de suas superintendências estaduais (BRASIL, 2008) ou através de parcerias com outras instituições públicas ou privadas. $\mathrm{O}$ recebimento dos animais nos CETAS pode ser classificado, de acordo com a procedência, em três formas distintas: a) apreensão, representada pelos animais decorrentes da ação fiscalizatória do IBAMA ou da Polícia Ambiental; b) recolhimento, resultado da captura de animais pelo IBAMA ou Polícia Ambiental; c) entrega voluntária, feita pelo cidadão que mantinha ilegalmente sob sua guarda animais silvestres (PAGANO et al., 2009).

A presente pesquisa objetivou diagnosticar, de acordo com as origens e as procedências dos animais, as espécies da fauna silvestre recebidas no CETAS de Belo Horizonte (BH), em 2011.

\section{MATERIAL E MÉTODOS}

Foi realizado um estudo epidemiológico observacional retrospectivo, baseado na análise exploratória das fichas de recebimento de animais silvestres do CETAS-BH, durante o período de janeiro a dezembro de 2011. O CETAS está localizado em Belo Horizonte, Minas Gerais e, no estado, é um dos responsáveis pelo recebimento dos animais apreendidos pela Polícia Militar do Meio Ambiente (PM MAMB) e pelo IBAMA e, igualmente, responsável pelos animais entregues voluntariamente por particulares a esses organismos.

As variáveis analisadas foram: espécies, número de espécimes, procedência (apreensão ou entrega voluntária), município em que os animais se encontravam (coordenadas geográficas) e data de entrada. Os dados originários das fichas alimentaram o programa EpiInfo 3.5.1 e a análise descritiva, incluindo a elaboração das tabelas e das distribuições de frequências que foram executados mediante o auxílio do software Microsoft Excel ${ }^{\circledR} 2010$.

Para a análise espacial, utilizaram-se as coordenadas geográficas da procedência dos animais, que foram obtidas através dos endereços presentes nas fichas de recebimento. Quando o endereço não estava disponível, utilizou-se a coordenada do centroide do município. Através das ferramentas de estatística espacial do software ArcGIS 9.3, determinaramse a média central, o evento central e a distribuição direcional para identificar as tendências na distribuição e as áreas de maior concentração dos eventos. Para a elipse de distribuição direcional, utilizou-se a distância de um desvio padrão (SANTOS \& JUNIOR, 2006). A análise espacial mais detalhada foi aplicada sobre a área identificada com a maior concentração dos eventos e assim foram determinadas com a função de Kernel; mapas ponderados para a quantidade de animais e o número de espécies de aves, répteis e mamíferos foram produzidos.

Para a identificação das espécies, foram consultados o Comitê Brasileiro de Registros Ornitológicos, CBRO (2014), a Lista Brasileira de Répteis (BÉRNILS \& COSTA, 2012) e o Catalogue of life (ROSKOV et al., 2013).

\section{RESULTADOS E DISCUSSÃO}

No ano de 2011, foram recebidos no CETAS-BH 7.426 animais vivos, registrados em 1.705 fichas. Os animais recebidos procediam de apreensões $(1.245,82,7 \%)$ e de entregas voluntárias $(460,17,3 \%)$. Foi registrada a entrada de 98 animais mortos. De acordo com BATISTA (2013 - Informe Verbal), a soltura foi a principal destinação (60\%) dos animais recebidos no CETAS-BH em 2011. Cerca de $10 \%$ dos animais permaneceram na instituição e outros $10 \%$ foram enviados para criatórios e zoológicos. A porcentagem de óbitos no período foi de $20 \%$.

Espécies da classe das aves representaram o maior número de recebimentos, 6.793 (91,5\%), enquanto os répteis e os mamíferos representaram apenas $522(7 \%)$ e $111(1,5 \%)$, respectivamente. A predominância das aves revela uma importante preferência por este grupo no tráfico de animais silvestres. Resultados semelhantes foram encontrados no $1^{\circ}$ Relatório Nacional sobre o Tráfico da Fauna Silvestre (RENCTAS, 2001a), por PAGANO et al. (2009), em pesquisa na Paraíba e por MOURA et al. (2012), em estudo realizado no CETAS de Piauí. 
Do total de 166 táxons recebidos no CETAS$\mathrm{BH}, 132(79,5 \%)$ pertenciam às aves, $16(9,65 \%)$ aos répteis e $18(10,85 \%)$ aos mamíferos. Observou-se maior variedade de táxons se comparado às pesquisas realizadas por MOURA et al. (2012), em que foram identificadas 108 táxons recebidos durante um ano no CETAS de Piauí, e por BASTOS et al. (2008), em que foram identificadas 130 táxons na avaliação dos animais recebidos de 1997 a 2005 em Goiás.

A principal procedência da maioria das aves foi a apreensão $(89,5 \%)$. Em contraste, a entrega voluntária foi mais expressiva para os répteis $(90,4 \%)$ e mamíferos (73,2\%). A PM MAMB foi responsável por $985(79,1 \%)$ ações de apreensão, com 4.952 $(80,6 \%)$ animais apreendidos. Os principais motivos das apreensões foram o patrulhamento ambiental e as denúncias anônimas de que havia animais silvestres em cativeiro. Segundo BORGES et al. (2006), as denúncias anônimas podem identificar a sensibilização da população frente ao tráfico.

As aves estavam distribuídas em 17 ordens e 34 famílias, (Tabela 1). A maioria, 83,7\% (5.686/6.793), pertencia à ordem Passeriformes e dela foram diagnosticadas 16 famílias (Tabela 2). A família mais representativa foi a Thraupidae (4.546, $79,6 \%$ ). Os resultados acima foram semelhantes aos encontrados por PAGANO et al. (2009) e DESTRO et al. (2012), em que os Passeriformes foram os mais frequentes nos recebimentos de seus estudos. Essa predominância é atribuída ao grande número de espécies e famílias e pelo fato de possuírem belo canto. Psittaciformes foi a segunda ordem com maior percentual de recebimentos $(880,13 \%)$, representados apenas pela família Psittacidae. As outras 15 ordens (Tabela 1) corresponderam a 2,5\% (171) do recebimento. Foram contabilizados ao número total de aves $56(0,82 \%)$ espécimes que foram identificadas apenas como aves.

Da família Thraupidae, foram recebidas 41 espécies, cujas principais foram: Sicalis flaveola, 1.323/6.793 (19,6\%), Saltator similis, 1.315 (19,4\%), Sporophila nigricollis, 710 (10,5\%) e Sporophila caerulescens, 454 (6,7\%). Na pesquisa realizada por DESTRO et al. (2012), as espécies Sicalis flaveola e Saltator similis também foram as mais recebidas no Brasil entre 2005 e 2009. Embora a abrangência temporal das pesquisas seja bastante diferente, esses resultados indicam a preferência entre criadores de pássaros pela captura ilegal dessas espécies.

Dezoito espécies de aves recebidas foram diagnosticadas como pertencentes à família Psittacidae (880 espécimes). As principais espécies foram Amazona aestiva, com 360/880 (41\%) indivíduos, e Psittacara leucophthalmus, com 232 (26,4\%) espécimes. Segundo RIBEIRO \& SILVA (2007), há grande preferência por esta família, devido à exuberante cor e beleza dos indivíduos, além da capacidade relativa que possuem de imitar a voz humana.

Os 522 répteis entregues ao CETAS$\mathrm{BH}$ pertenciam a três ordens e 11 famílias distintas (Tabela 1). A ordem Squamata estava representada por $66,3 \%(346 / 522)$ animais, distribuídos em seis famílias, das quais a principal foi a família Boidae , com três espécies: Boa constrictor com 62/346 (17,9\%), Epicrates cenchria, 262 (75,7\%), e Eunectes murinus com apenas um exemplar. A maioria dos exemplares da família Boidae $(318,98 \%)$ originou da entrega voluntária, realizada por um mesmo autor, após o encerramento de um criatório desses animais. Os demais exemplares dessa família, 7 (2\%), eram da espécie Boa constrictor e podem estar associados à criação em cativeiro ilegal, com a finalidade de estimação, já que os seres humanos são atraídos pela beleza e a menor necessidade de atenção, referentes à alimentação e ao alojamento, conforme descrito por SHIAU et al. (2006). Já a ordem Testudines, durante o período de estudo, esteve representada por 173/522 (33\%) espécies, distribuídas em quatro famílias distintas.

$\mathrm{O}$ pequeno grupo de mamíferos recebidos, 111 animais, estava distribuído em sete ordens e 14 famílias (Tabela 1). A ordem dos Primatas representou $31,5 \%$ dos recebimentos com 35 exemplares, de quatro famílias diferentes. A família Callitrichidae apresentou 31 (28\%) animais recebidos, de duas espécies: Callithrix penicillata, 30 espécimes, e $\boldsymbol{C}$. geoffroyi, com apenas um exemplar. A captura e a criação ilegal de espécimes silvestres representam riscos à saúde humana, pois eles podem ser reservatórios e transmissores de zoonoses ao homem e aos animais domésticos (MATZ-RENSING et al., 2003).

Das 166 espécies de animais silvestres identificadas no ano de 2011, 24 (15\%) estão sob algum tipo de ameaça. O status de conservação dessas espécies de animais silvestres recebidos no CETASBH em 2011 pode ser observado nas Tabelas 1 e 2.

As apreensões e as entregas voluntárias de animais silvestres apresentaram-se dispersas por todo o Estado de Minas Gerais (Figura 1). Os registros de recebimentos incluíram 94 municípios, desses, 30 pertencem à Região Metropolitana de Belo Horizonte (RMBH). Dos animais recebidos, $75 \%$ (5.589) eram provenientes dessa região, sendo os principais municípios detentores de fauna: Belo Horizonte (30\%), Contagem $(9,7 \%)$, Betim $(8,3 \%)$ e Ibirité $(4,1 \%)$. Houve uma perda de informação de 
Tabela 1 - Classificação das aves ${ }^{1}$, répteis e mamíferos recebidos no CETAS-BH, 2011.

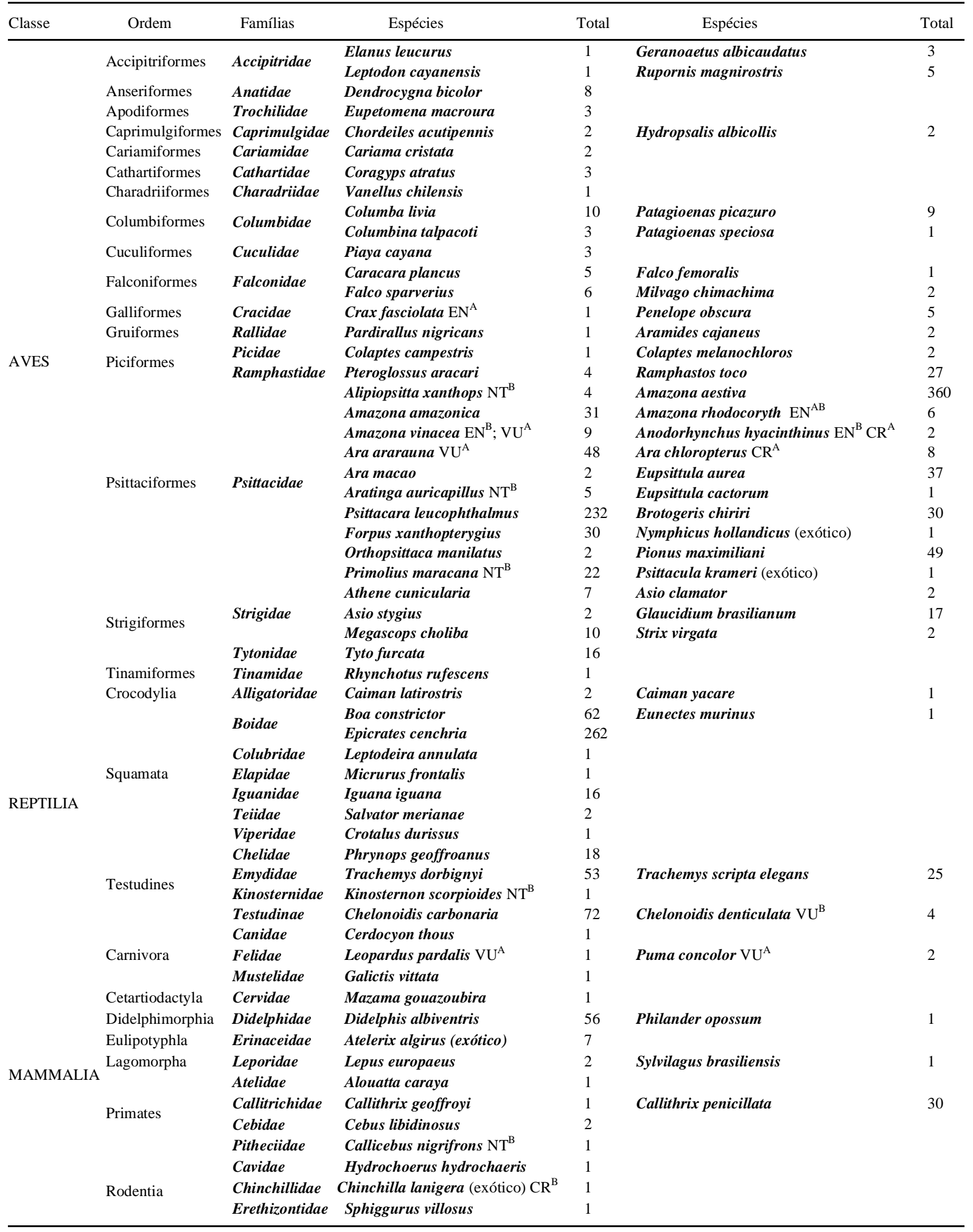

${ }^{1} \mathrm{~A}$ ordem Passeriformes, da classe aves, está detalhada na tabela 2.

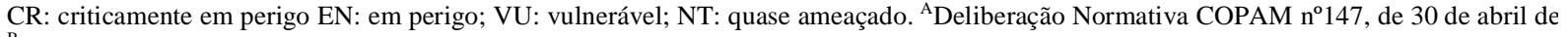
${ }^{\mathrm{B}}$ International Union for Conservation of Nature. Red List of Threatened Species. Version 2013.1.

Ciência Rural, v.45, n.1, jan, 2015. 
Tabela 2 - Classificação das aves da ordem Passeriformes recebidas no CETAS-BH, 2011.

\begin{tabular}{|c|c|c|c|c|}
\hline Famílias & Espécies & Total & Espécies & Total \\
\hline Cardinalidae & Cyanoloxia brissonii & 221 & & \\
\hline Corvidae & Cyanocorax cristatellus & 1 & & \\
\hline Cotinginae & Procnias nudicollis $\mathrm{VU}^{\mathrm{B}} ; \mathrm{EN}^{\mathrm{A}}$ & 4 & & \\
\hline \multirow[t]{21}{*}{ Thraupidae } & Cissopis leverianus & 2 & Dacnis cayana & 8 \\
\hline & Hemithraupis ruficapilla & 2 & Lanio cucullatus & 1 \\
\hline & Lanio pileatus & 90 & Lophospingus pusillus (exótico) & 2 \\
\hline & Paroaria coronata & 13 & Neothraupis fasciata $\mathrm{NT}^{\mathrm{B}}$ & 1 \\
\hline & Ramphocelus bresilius & 2 & Paroaria dominicana & 23 \\
\hline & Saltatricula atricollis & 20 & Saltator similis & 1315 \\
\hline & Schistochlamys ruficapillus & 47 & Schistochlamys melanopis & 5 \\
\hline & Sicalis columbiana & 2 & Sicalis flaveola & 1323 \\
\hline & Sicalis luteola & 6 & Sporophila albogularis & 23 \\
\hline & Sporophila angolensis $\mathrm{CR}^{\mathrm{A}}$ & 108 & Sporophila bouvreuil & 11 \\
\hline & Sporophila caerulescens & 454 & Sporophila collaris & 4 \\
\hline & Sporophila falcirostris $\mathrm{VU}^{\mathrm{B}} ; \mathrm{EN}^{\mathrm{A}}$ & 5 & Sporophila frontalis $\mathrm{VU}^{\mathrm{B}} ; \mathrm{EN}^{\mathrm{A}}$ & 29 \\
\hline & Sporophila leucoptera & 8 & Sporophila lineola & 100 \\
\hline & Sporophila maximiliani $\mathrm{NT}^{\mathrm{B}} ; \mathrm{CR}^{\mathrm{A}}$ & 90 & Sporophila nigricollis & 710 \\
\hline & Sporophila palustris $\mathrm{EN}^{\mathrm{B}} ; \mathrm{CR}^{\mathrm{A}}$ & 1 & Sporophila plumbea & 8 \\
\hline & Tangara cayana & 7 & Tachyphonus coronatus & 2 \\
\hline & Tangara cyanoventris & 6 & Tangara cyanocephala & 2 \\
\hline & Tangara ornata & 1 & Tangara episcopus & 1 \\
\hline & Tangara sayaca & 48 & Tangara palmarum & 12 \\
\hline & Tersina viridis & 13 & Tiaris fuliginosus & 1 \\
\hline & Volatinia jacarina & 40 & & \\
\hline \multirow[t]{2}{*}{ Estrildidae } & Amadina fasciata (exótico) & 2 & Erythrura psittacea (exótico) & 1 \\
\hline & Estrilda astrild & 6 & & \\
\hline \multirow[t]{4}{*}{ Fringillidae } & Carduelis carduelis & 1 & Euphonia chalybea $\mathrm{NT}^{\mathrm{B}} ; \mathrm{EN}^{\mathrm{A}}$ & 6 \\
\hline & Euphonia chlorotica & 4 & Euphonia violacea & 3 \\
\hline & Serinus canaria (exótico) & 7 & Sporagra magellanica & 94 \\
\hline & Sporagra yarrellii $\mathrm{VU}^{\mathrm{B}}$ & 4 & & \\
\hline Furnariidae & Furnarius rufus & 1 & & \\
\hline Hirundinidae & Pygochelidon cyanoleuca & 10 & & \\
\hline \multirow[t]{4}{*}{ Icteridae } & Agelasticus thilius & 1 & Chrysomus ruficapillus & 16 \\
\hline & Gnorimopsar chopi & 188 & Icterus jamacaii & 30 \\
\hline & Molothrus bonariensis & 10 & Psarocolius decumanus & 1 \\
\hline & Pseudoleistes guirahuro & 1 & & \\
\hline Mimidae & Mimus saturninus & 3 & & \\
\hline Passeridae & Passer domesticus & 3 & & \\
\hline Passerillidae & Zonotrichia capensis & 241 & & \\
\hline Pipridae & Chiroxiphia caudata & 1 & Ilicura militaris & 1 \\
\hline Sturnidae & Sturnus vulgaris (exótico) & 2 & & \\
\hline \multirow[t]{3}{*}{ Turdidae } & Turdus albicollis & 8 & Turdus amaurochalinus & 72 \\
\hline & Turdus fumigatus & 2 & Turdus leucomelas & 87 \\
\hline & Turdus rufiventris & 93 & & \\
\hline Tyrannidae & Elaenia flavogaster & 1 & Pitangus sulphuratus & 14 \\
\hline
\end{tabular}

CR: criticamente em perigo EN: em perigo; VU: vulnerável; NT: quase ameaçado.

${ }^{\mathrm{A}}$ Deliberação Normativa COPAM n ${ }^{\circ} 147$, de 30 de abril de 2010;

${ }^{\mathrm{B}}$ International Union for Conservation of Nature. Red List of Threatened Species. Version 2013.1.

$325(4,5 \%)$ animais, os quais não continham em seus registros a localidade de procedência.

Técnicas espaciais descritivas verificaram a importância da $\mathrm{RMBH}$ e de áreas vizinhas no tráfico da fauna silvestre (Figura 1). A média central identificou a coordenada central entre as distâncias de todos os dados georreferenciados, o evento central determinou a ocorrência mais próxima a esse ponto

Ciência Rural, v.45, n.1, jan, 2015. 


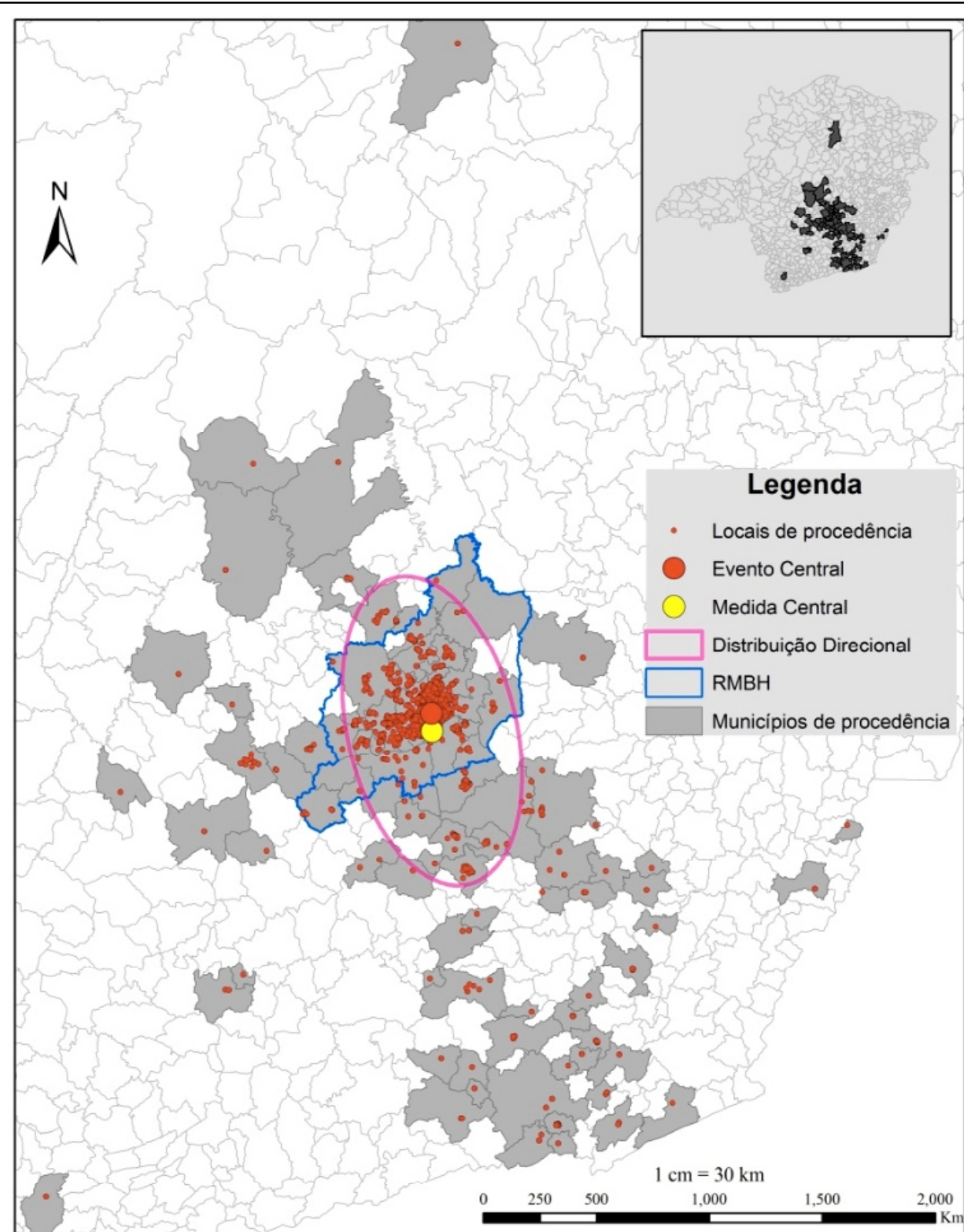

Figura 1 - Distribuição espacial dos animais recebidos no centro de triagem de animais silvestres de Belo Horizonte, CETAS-BH, 2011

central; os dois em Belo Horizonte. A distribuição direcional delimitou a área de maior concentração das ocorrências, a elipse envolveu $81,5 \%$ de todos os animais avaliados neste estudo, 37 municípios foram intersectados, 26 pertencentes à RMBH; identificouse um sentido direcional do noroeste ao sudeste.

Os mapas de densidade de Kernel (Figura 2) para as espécies de aves (A), mamíferos (B) e répteis (C) identificaram áreas de maior densidade na RMBH. A classe com maior concentração foram as aves, envolvendo principalmente e em toda sua extensão, o município de Belo Horizonte. O resultado foi similar para o total de animais (D, E e F). No caso das aves, foi identificada uma concentração de até 16,43 aves $\mathrm{km}^{-2}$.

$\mathrm{O}$ conhecimento da fauna oriunda de captura, do comércio e da manutenção ilegais no estado é ferramenta importante para a conservação das espécies, pois permite o aprimoramento de ações educadoras socioambientais, dirigidas à população,

Ciência Rural, v.45, n.1, jan, 2015. 


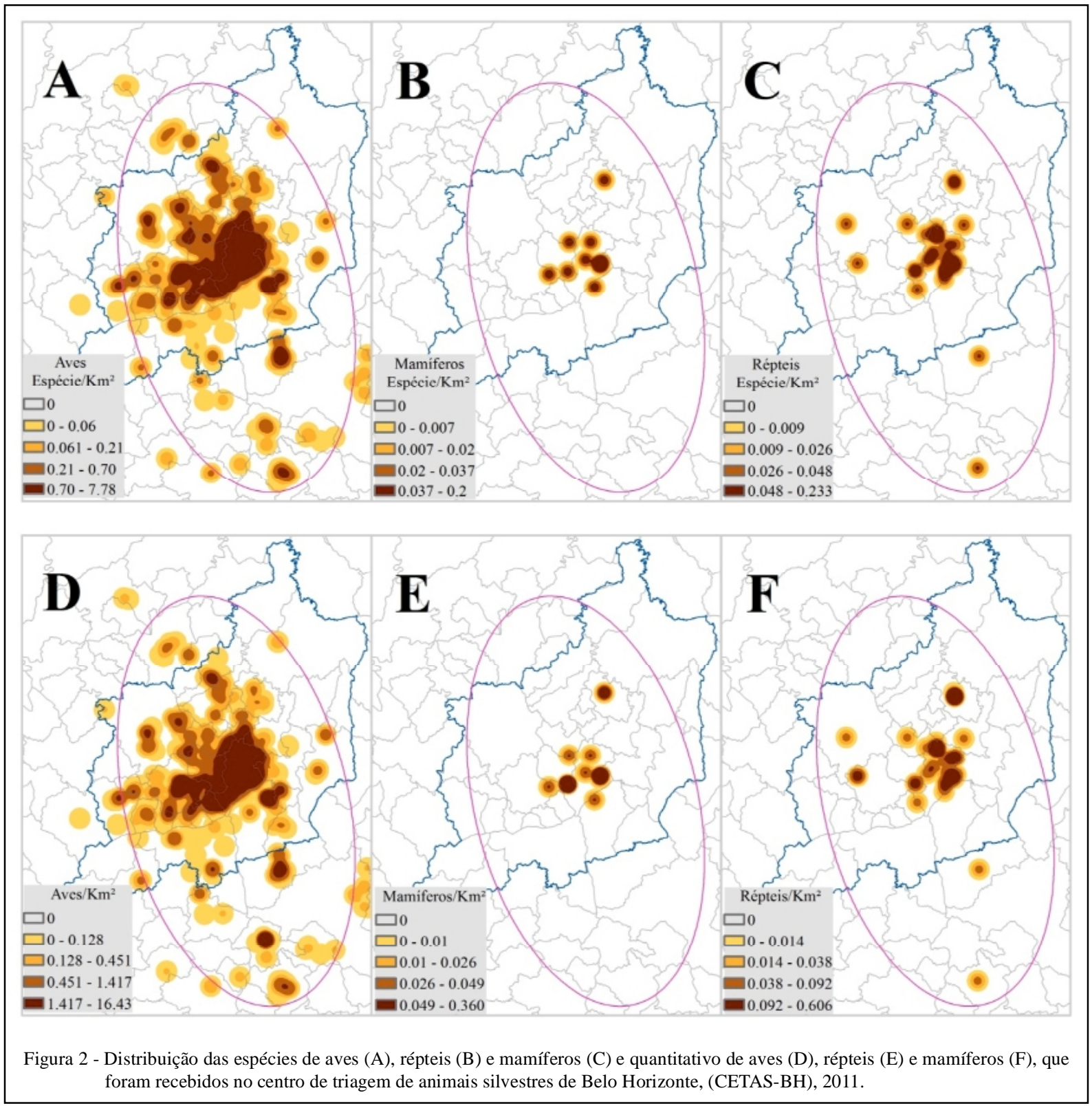

e ações fiscalizadoras estrategicamente localizadas em relação ao tráfico de animais silvestres. Esses resultados permitem observar a grande variedade e quantidade de animais afetados pelo tráfico em um dos principais centros urbanos do país. Essa mesma região já foi destacada por RENCTAS (2001b) como importante local de venda de animais, participando assim da rota do tráfico de animais silvestres em Minas Gerais. Deve-se ressaltar também que a localização do CETAS-BH nessa região é um fator importante para esta avaliação, já que a proximidade facilita o encaminhamento desses animais para a instituição, o que pode ser uma das causas do expressivo volume recebido. Também é válido frisar que o menor número de animais procedentes dos outros municípios não significa uma redução do tráfico de silvestres nesses locais, pois o fato pode estar associado a ações fiscalizatórias menos intensas.

\section{CONCLUSÃO}

O CETAS-BH recebeu no ano de 2011 uma grande quantidade de animais, de diversos táxons. A apreensão realizada pela PM MAMB foi a principal procedência de entrada desses espécimes, sendo que a maioria era proveniente da RMBH, que 
foi identificada como principal destino dos animais comercializados ilegalmente em MG. As aves foram o grupo predominante em número. As espécies Sicalis flaveola e Saltator similis representaram juntas mais de um terço dos animais recebidos e, apesar de não ameaçadas, sofreram grande pressão de captura. Os répteis e mamíferos foram recebidos em menor número e principalmente por entrega voluntária. Mais de $15 \%$ das espécies recebidas no CETASBH encontraram-se sob algum tipo de ameaça. Esses resultados são úteis na elaboração de ações de caráter educativo-ambiental, direcionadas para cada município, de forma mais ágil e localizada.

\section{COMITÊ DE ÉTICA}

Declaro, para os devidos fins, que o artigo intitulado "Distribution of wildlife trafficking in Minas Gerais, 2011", não foi submetido para avaliação ao Camitê de Ética da Universidade Federal de Minas Gerais. Desta forma, os autores assumem total responsabilidade pelos dados apresentados e estão disponíveis para possíveis questionamentos, caso venham a ser requeridos.

\section{INFORME VERBAL}

BH, dezembro de 2011.

BATISTA, M. Relatório anual consolidado CETAS-

\section{AGRADECIMENTOS}

Ao CETAS-BH, a Escola de Veterinária da Universidade Federal de Minas Gerais e a Coordenação de Aperfeiçoamento de Pessoal de Nível Superior (CAPES).

\section{REFERÊNCIAS}

BASTOS, L.F. et al. Apreensão de espécimes da fauna silvestre em Goiás: situação e destinação. Revista de Biologia Neotropical, v.5, p.51-63, 2008. Disponível em: <http://www.revistas.ufg.br/ index.php/RBN/article/view/9822/6707>. Acesso em: 2 jun. 2013.

BÉRNILS, R.S.; COSTA, H.C. (Org.). Répteis brasileiros: lista de espécie. Versão 2012.2. Sociedade Brasileira de Herpetologia, 2012. Disponível em: 〈http://www.sbherpetologia.org.br〉. Acesso em: 14 jun. 2013.

BIODIVERSITAS, Revisão das listas das espécies da flora e da fauna ameaçadas de extinção do Estado de Minas Gerais, 2007. Disponível em: <http://www.biodiversitas.org.br/listas-mg/>. Acesso em: 14 jun. 2013.

BORGES, R.C. et al. Diagnóstico da fauna silvestre apreendida e recolhida pela Polícia Militar de Meio Ambiente de Juiz de Fora, MG (1998 e 1999). Revista Brasileira Zootecnia, v.8, p.23, 2006.
BRASIL, Instrução Normativa n.169, de 20 de fevereiro de 2008. Ministério do Meio Ambiente, Brasília, DF, p.13. Disponível em: <http://www.mma.gov.br/port/conama/legiabre. cfm?codlegi=585>. Acesso em: 1 jul. 2013.

CBRO (COMITÊ BRASILEIRO DE REGISTROS ORNITOLÓGICOS). Listas das aves do Brasil. 11.ed. 2014. Disponível em: <http://www.cbro.org.br>. Acesso em: 06 jan. 2014.

DESTRO, G.F.G. et al. Efforts to combat wild animals trafficking in Brazil. In: Biodiversity enrichment in a diverse world, 2012. Chapter XX. Disponível em: <http://www.ibama.gov.br/sophia/ cnia/periodico/esforcosparaocombateaotraficodeanimais.pdf $>$. Acesso em: 15 jun. 2013.

MATZ-RENSING, K. et al. Fatal Herpes simplex infection in a group of common marmosets (Callithrix jacchus). Veterinary Pathology, v.40, n.4, p.405-411, 2003.

MOURA, S.G. et al. Animais silvestres recebidos pelo centro de triagem do IBAMA no Piauí no ano de 2011. Enciclopédia: Biosfera, Goiânia, v.8;p.1762, 2012.

PAGANO, I.S.A. et al. Aves depositadas no Centro de Triagem de Animais Silvestres do IBAMA na Paraíba: uma amostra do tráfico de aves silvestres no estado. Ornithologia,v.3, n.2, p.132-144, 2009.

RENCTAS (REDE NACIONAL CONTRA O TRÁFICO DE ANIMAIS SILVESTRES). $\mathbf{1}^{\circ}$ Relatório Nacional sobre o tráfico de fauna silvestre, 2001a. 107p. Disponível em: <www.renctas.com.br/ files/REL_RENCTAS_pt_final.pdf>. Acesso em: 01 jul. 2012.

RENCTAS (REDE NACIONAL CONTRA O TRÁFICO DE ANIMAIS SILVESTRES). Principais rotas terrestres utilizadas para o tráfico de animais silvestres Brasil - região Sudeste, 2001b. Disponível em: <http://www.renctas.org.br/>. Acesso em: 01 jul. 2013

RIBEIRO, L.B.; SILVA, M.G. O comércio ilegal põe em risco a diversidade das aves no Brasil. Ciência e Cultura. [online], v.59, n.4, p.4-5, 2007.

ROSKOV, Y. et al. Species 2000 \& ITIS Catalogue of Life, 2013 Annual Checklist. Disponível em: $<$ http//www.catalogueoflife. org>. Acesso em: 23 maio, 2013.

SANTOS, L; JUNIOR, A.A.R. Distribuição espacial dos acidentes de trânsito em São Carlos (SP): identificação de tendência de deslocamento através da técnica de elipse de desvio padrão. Caminhos de Geografia, v.7, n.18, p.1-7, 2006.

SANTOS, T.C.C.; CÂMARA, J.B.D. GeoBrasil 2002: perspectivas do meio ambiente no Brasil - o estado da biodiversidade. Brasília: IBAMA, 2002. p.447.

SHIAU, T.W. et al. A survey on alien pet reptiles in Taiwan. Taiwania, v.51, n.2, p.71-80, 2006. Disponível em: <http://tai2. ntu.edu.tw/taiwania/pdf/tai.2006.51.2.71.pdf $>$. Acesso em: 1 ago. 2013. 